\title{
Serotonin and tryptophan metabolites, autoantibodies and gut microbiome in APECED
}

\author{
Emmi Naskali ${ }^{1}$, Katja Dettmer ${ }^{2}$, Peter J Oefner ${ }^{2}$, Pedro A B Pereira ${ }^{3}$, Kai Krohn ${ }^{4}$, Petri Auvinen ${ }^{3}$, Annamari Ranki ${ }^{1}$ \\ and Nicolas Kluger ${ }^{1}$
}

1'Department of Dermatology, Allergology and Venereology, University of Helsinki, and Helsinki University Central Hospital, Helsinki, Finland ${ }^{2}$ Institute of Functional Genomics, University of Regensburg, Regensburg, Germany

${ }^{3}$ Institute of Biotechnology, DNA Sequencing and Genomics Laboratory, University of Helsinki, Helsinki, Finland

${ }^{4}$ Clinical Research Institute HUCH Ltd, Biomedicum Helsinki 1, Helsinki, Finland

Correspondence should be addressed to N Kluger: nicolas.kluger@hus.fi

\begin{abstract}
Objective: Intestinal autoimmunity with gastrointestinal (Gl) dysfunction has been shown in patients with autoimmune polyendocrinopathy-candidiasis-ectodermal dystrophy (APECED). Patients lack entero-endocrine (EE) cells and have circulating autoantibodies (Aabs) against critical enzymes in serotonin (5-HT) biosynthesis.

Design: We sought to determine the serum levels of 5-HT, tryptophan (Trp) metabolites and L-DOPA in 37 Finnish APECED patients and to correlate their abundance with the presence of TPH and AADC Aabs, Gl dysfunction and depressive symptoms. We also performed an exploratory analysis of the gut microbiome.

Methods: Serum 5-HT, L-DOPA and Trp metabolite levels were determined by liquid chromatography-electrospray ionization-tandem mass spectrometry (LC-ESI-MS/MS). TPH and AADC Aabs were measured by ELISA. Depression was assessed with a structured RBDI questionnaire. The V3-V4 regions of the bacterial $16 \mathrm{~S}$ rRNA gene were sequenced for gut microbiome exploration.

Results: Serum 5 -HT levels were significantly decreased $(130 \pm 131 \mathrm{nmol} / \mathrm{L}$ vs $686 \pm 233 \mathrm{nmol} / \mathrm{L}, P<0.0001$ ) in APECED patients with TPH-1 ( \pm AADC) Aabs compared to controls and patients with only AADC Aabs. Reduced 5-HT levels correlated with constipation. The genus Escherichia/Shigella was overrepresented in the intestinal microbiome. No correlation between serum Trp, 5-HT or L-DOPA levels and the RBDI total score, fatigue or sleep disorders was found.

Conclusions: This exploratory study found low serum levels of 5-HT to be associated with constipation and the presence of TPH-1 and AADC Aabs, but not with symptoms of depression. Hence, serum 5-HT, TPH1 and AADC Aabs should be determined in APECED patients presenting with $\mathrm{Gl}$ symptoms.
\end{abstract}
Key Words
- APECED
- serotonin
- diarrhea
- constipation
- entero-endrocrine cells
- Paneth cells

Endocrine Connections (2019) 8, 69-77

\section{Introduction}

Autoimmune polyendocrinopathy-candidiasisectodermal dystrophy (APECED, OMIM 240300) or autoimmune polyendocrine syndrome type 1 (APS-1) is a rare autosomal recessive disease caused by a loss-offunction mutation of the AIRE (Autoimmune Regulator) gene located on chromosome 21 (21q22.3) (1, 2, 3, 4).
The disease predisposes to chronic mucocutaneous candidiasis and various autoimmune disorders that impair quality of life (1). AIRE is normally expressed in thymus, where it is responsible for the elimination of potentially autoreactive cytotoxic T-cells, which in APECED will destroy target cells. Due to the loss of AIRE function, https://ec.bioscientifica.com

https://doi.org/10.1530/EC-18-0513

(c) 2019 The authors Published by Bioscientifica Ltd

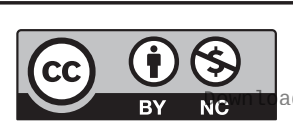

This work is licensed under a Creative Commons Attribution-NonCommercial 4.0 International License. ded from Bioscientifica.com at $04 / 26 / 2023$ 12:52:44PM 
APECED patients also have a lack of regulatory T-cells and, thus, APECED is characterized by a variety of hightiter circulating autoantibodies (Aabs). Organ- specific antibodies in APECED do not cause tissue destruction per $s e$, but rather indicate the destructive action of cytotoxic T-cells. The most common non-organ-specific Aabs are neutralizing Aabs against type I interferon (IFN- $\alpha$ and - $\omega)$ and Th17 cytokines (IL-17A, IL-17F, IL-22). Patients also display Aabs directed against intracellular enzymes involved in hormone and neurotransmitter biosynthesis $(5,6)$. Among the latter, Aabs directed against aromatic L-amino acid decarboxylase (or DOPA decarboxylase, AADC) and tryptophan hydroxylase (TPH) are commonly found in APECED patients $(7,8,9)$ and their presence has been associated with the lack of entero-endocrine (EE) cells of the gastrointestinal (GI) tract $(9,10)$. Serotonin (5-hydroxytryptamine, 5-HT) is synthesized mainly (95\%) by GI EE cells and in smaller amounts in the central nervous system (11). Its synthesis is derived from tryptophan (Trp) in a two-step reaction, involving TPH-1 or TPH-2 and AADC. AADC catalyzes both the decarboxylation of 5-hydroxytryptophan to 5-HT and the conversion of L-DOPA to dopamine in the brain. 5-HT is an important biogenic amine and neurotransmitter that affects intestinal motility as well as mood, sleep and appetite (11). APECED patients with circulating TPH-1 Aabs have been shown to have significantly lower serum levels of 5-HT than patients without such Aabs (12). We have previously reported that severe constipation significantly correlated with TPH-1 and/or AADC Aabs and with lacking 5-HT expression in GI EE cells (9). Both 5-HT and dopamine are known to be related to the mental balance of an individual, and it is of interest to note that we have found depressive symptoms in $29 \%$ of Finnish APECED patients (13).

Besides, alteration of gut microbiome composition has been shown to be associated with various diseases such as mental depression and autoimmune diseases (14). It has become evident that gut microbes may directly influence the central nervous system function by controlling the production of neurotransmitters, such as 5 -HT in the gut (15).

In the present study, we assessed the serum levels of 5-HT, L-DOPA and Trp metabolites in our cohort of Finnish APECED patients and compared the results with the presence of TPH-1, TPH-2 and AADC Aabs and with clinical manifestations such as GI dysfunction and depressive symptoms. We also compared the gut microbiota of APECED patients against that of healthy control persons to assess whether there were any indications of potential microbiome differences between the two populations.

\section{Materials and methods}

\section{Design and patients}

We recruited 37 Finnish APECED patients with confirmed AIRE mutations (25 women and 12 men, sex ratio 2.1:1). As the Finnish patient group has a history of willingly participating in research of their disease, the study recruitment and contact information was collected with the help of the APECED patient's association (APECED ja Addison Ry, http://www.apeced.org). Most of these patients have been included in various publications on APECED in Finland $(1,13)$, including our previous work about gastrointestinal autoimmunity (9). The mean age of the cohort was 39.9 years (range 9-70 years), including five children aged between 9 and 16 years with a mean age of 12.4 years. The study was approved by the Ethics Committee of Medicine, Helsinki and Uusimaa Joint Authority (HUS 1127/2016). Each participant (or their parents if under the age of 18 years) signed an informed consent and permission to review all their hospital records. For serum metabolite analysis, an additional 12 healthy, age- and sex-matched volunteers (control persons) participated, after a 24-hour long serotonin-free diet (see below). The control group had no features of APECED or any other autoimmune disease, no GI symptoms, and nobody was on an antidepressant medication.

\section{Questionnaire}

The Raitasalo's modification of the Beck Depression Inventory (RBDI) mood questionnaire is a self-reported questionnaire that has been used in Finland for the past 30 years for screening and grading depression. This modified Finnish version of Beck's 13-item depression scale has 13 questions for depression. Depression is measured on the basis of 13 items scoring from 0 to 3, and score ranges from zero to 39 points. Five to seven points refer to mild depression, 8 to 15 points to moderate depression and over 16 points to severe depression. We also included two questions regarding gastrointestinal function, that is, current chronic constipation or diarrhea (without any known cause) or both.

The questionnaire was sent to all 37 patients. Twentysix patients completed and returned the questionnaires (response rate 70.3\%). One questionnaire was anonymous and one patient answered only the GI-related questions.

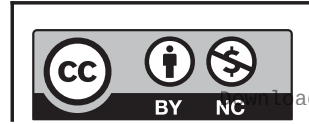

This work is licensed under a Creative Commons Attribution-NonCommercial 4.0 International License. ded from Bioscientifica.com at 04/26/2023 12:52:44PM 


\section{Assay of autoantibodies}

Serum circulating Aabs against recombinant AADC (Biosite, Täby, Sweden), TPH-1 (Sino Biological Inc., Beijing, China) and TPH-2 (OriGene Technologies Inc. Rockville, MD, USA) were assayed with a validated in-house ELISA as previously described in detail (9). Sera from healthy blood donors obtained from the Finnish Red Cross blood service served as reference. The cut-off value was set as mean \pm 2 s.D. of the reference samples.

\section{Serum levels of 5-HT, L-DOPA and Trp metabolites}

Of the 37 patients, 26 patients (16 women and 10 men, participation rate $70.2 \%$ ) and 12 healthy, age- and sexmatched volunteers participated in the measurement of serum metabolite levels. The sera were drawn after an overnight fasting and a 24-hour long serotonin-free diet, refraining patients and controls from eating anything that might include serotonin or its precursor such as nuts, banana, avocado, plum, kiwi, pineapple, grape, melons, date, tomato, eggplant and blue cheese. The venous blood specimens $(5 \mathrm{~mL})$ were collected in plastic anticoagulant gel-free vacuum tubes, sent at room temperature to our laboratory, where serum was separated by centrifugation and stored at $-80^{\circ} \mathrm{C}$. For the determination of $5-\mathrm{HT}$, L-DOPA and various Trp metabolites by liquid chromatography-electrospray ionization-tandem mass spectrometry (LC-ESI-MS/MS), the frozen serum samples were sent on dry ice to the Institute of Functional Genomics in Regensburg, Germany. LC-ESI-MS/MS analysis was performed as previously described (16) with the inclusion of L-DOPA. The latter elutes at $2.55 \mathrm{~min}$ and the transitions $\mathrm{m} / \mathrm{z} 198.2 \longrightarrow \mathrm{m} / \mathrm{z} 152$ for L-DOPA and $\mathrm{m} / \mathrm{z} 201.2 \longrightarrow \mathrm{m} / \mathrm{z} 155$ for the corresponding deuterated internal standard L-DOPA-d3 were monitored. Other metabolites determined included Trp and its metabolites 3-hydroxykynurenine (HK), nicotinamide (NAM), 5-HT, kynurenine (KYN), xanthurenic acid, kynurenic acid (KA), anthranilic acid (AA), 3-hydroxyanthranilic acid (HAA), 5-hydroxyindole-3-acetic acid (HIAA), indole-3-lactic acid (ILA), indole-3-acetic acid (IAA) and indole-3-propionic acid (IPA).

\section{Gut microbiome}

Stool specimens for $16 \mathrm{~S}$ rRNA-based gut microbiome composition analysis were collected by ten Finnish APECED patients (aged 17-58, seven females) and eight healthy Finnish volunteers (aged 16-77, 5 females) at home using collection kits preloaded with DNA stabilizer (PSP Spin Stool DNA Plus Kit: Stratec Molecular GmbH, Berlin, Germany). The tubes were then kept in the subject's home freezer until the clinical visit, after which they were stored at $-80^{\circ} \mathrm{C}$. DNA extraction was performed with the QIAamp Fast DNA Stool Mini Kit, Cat No. 51604 (Qiagen), according to the manufacturer's instructions. PCR amplification (two-step), quantification, pooling, purification and pool quality assessment of the bacterial 16S rRNA gene were performed according to a previously published protocol (17), except that in the present study, we amplified the V3-V4 regions. One PCR blank (no template) was also sequenced to assess potential contamination. For amplification of these regions, we used a mixture of the 'universal' bacterial primers 341F1-4 (5' CCTACGGGNGGCWGCAG 3') and 785R1-4 (5' GACTACHVGGGTATCTAATCC 3'), with the following partial Illumina TruSeq adapter sequences added to their 5' ends:

F1: ATCTACACTCTTTCCCTACACGACGCTCTTCCGATCT, F2: ATCTACACTCTTTCCCTACACGACGCTCTTCCGA TCTgt,

F3: ATCTACACTCTTTCCCTACACGACGCTCTTCCGATC Tagag,

F4: ATCTACACTCTTTCCCTACACGACGCTCTTCCGATC Ttagtgt,

R1: GTGACTGGAGTTCAGACGTGTGCTCTTCCGATCT, R2: GTGACTGGAGTTCAGACGTGTGCTCTTCCGATCTa, R3: GTGACTGGAGTTCAGACGTGTGCTCTTCCGATCTtct, R4: GTGACTGGAGTTCAGACGTGTGCTCTTCCGATC Tctgagtg.

The small letters correspond to additional nucleotides that are added for mixing purposes during sequencing. The amplicon sequencing was performed with an Illumina MiSeq (v3 600 cycle kit), with 325 bases for the forward and 285 bases for the reverse read. DNA library creation and sequencing were performed at the DNA Sequencing and Genomics Laboratory of the University of Helsinki, Finland. The raw sequencing data amounted to $3,733,753$ sequences. FastQC (18) and cutadapt (19) were used to assess the sequence quality and to trim primers, respectively. The sequence data were then clustered into Operational Taxonomic Units (OTUs) and classified taxonomically with mother (20) following the Standard Operating Procedure for MiSeq available online (http://www.mothur.org/wiki/MiSeq_SOP). The final data set (after removal of the PCR blank) consisted of a total of 2,778,416 sequence reads representing 3,662 OTUs.

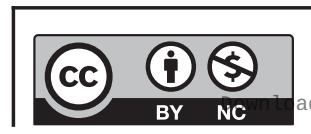


The largest amount of reads per sample was 153,653, and the lowest 85,508 , with a mean of 120,801 reads per sample.

\section{Statistical analysis}

Statistical analysis was conducted with SPSS Statistics 22 (SPSS Inc., an IBM company). Quantitative data were expressed as mean values \pm s.D. Comparisons of categorical variables were performed using the chi-square test. Serum metabolite levels between APECED patients and controls were compared by ANOVA with Tukey's and Dunnett's post hoc test. Correlation between RBDI scores and serum metabolite levels were analyzed by Spearman's rank correlation coefficient. A $P$ value of $<0.05$ was considered significant. All sequence data were imported into R (https://www.R-project.org/) and manipulated with the phyloseq package (21). Differential abundance of bacterial taxa was analyzed using DESeq2 (22), which is based on negative binomial linear models. Alpha diversity was analyzed with non-rarefied OTU-level data using the Shannon and inverse Simpson indices. Statistical tests were performed with the (unpaired) pairwise Wilcoxon ranksum test for comparisons between controls and APECED subjects. Beta diversity analyses used rarefied data at OTU-level, by performing Non-Metric Multidimensional Scaling (NMDS) on Bray-Curtis dissimilarity, as well as Permutational Multivariate Analysis of Variance Using Distance Matrices (adonis, from the vegan package (https://cran.r-project.org/web/packages/vegan/index. html)), an implementation of PERMANOVA. A doubletailed $P$ value of $<0.05$ was considered statistically significant for all microbiome-related analyses.

\section{Results}

\section{Altered 5-HT serum levels associate with autoantibodies against TPH-1 and AADC}

Circulating Aabs against AADC and TPH-1 were detected in $20(54 \%)$ and $15(40.5 \%)$ of the 37 APECED patients, respectively. Among the 26 patients for whom Trp metabolites analysis was performed, the distribution of Aabs was similar: 54\% $(n=14)$ had AADC Aabs and $46 \%$ $(n=12)$ had TPH-1 Aabs. None of the APECED patients had detectable Aabs against the brain TPH isoenzyme TPH-2. The mean ( \pm s.D.) serum 5-HT level was significantly decreased in APECED patients with TPH-1 ( \pm AADC) Aabs $(130 \pm 131 \mathrm{nmol} / \mathrm{L})$ compared to healthy controls and patients without Aabs or with only AADC Aabs $(686 \pm 233 \mathrm{nmol} / \mathrm{L})$. Although patients with both TPH-1 and AADC Aabs tended to have lower 5-HT serum levels $(105 \pm 78 \mathrm{nmol} / \mathrm{L})$ than patients with TPH-1 Aabs only $(217 \pm 214 \mathrm{nmol} / \mathrm{L})$, the difference was not statistically significant (Fig. 1, Table 1; Supplementary Table 1, see section on supplementary data given at the end of this article). The sole presence of circulating AADC Aabs did not affect 5-HT serum levels compared with controls and APECED patients without Aabs. TPH-1 Aabs and/or AADC Aabs did not associate with changes in serum levels of Trp or any of its metabolites other than 5-HT. Interestingly, patients with AADC Aabs alone or in association with TPH-1 Aabs had significantly higher serum levels of L-DOPA than controls (Table 1).

\section{Constipation symptoms correlate with lower 5-HT levels in APECED patients}

Four patients $(16 \%, 4 / 25)$ reported current and chronic diarrhea (1 male, 3 female), three $(15.3 \%, 3 / 25)$ reported chronic constipation (1 male, 2 female), while no one reported both symptoms to be simultaneous/ alternating at the time of the blood sampling. Mean 5-HT concentration was lower among patients reporting constipation compared to those without $(24 \pm 12 \mathrm{nmol} / \mathrm{L}$ vs $488 \pm 333 \mathrm{nmol} / \mathrm{L} ; P=0.027$ ) (Fig. 2). No significant association with any other metabolite was found. Diarrhea did not associate with any metabolite level variation.

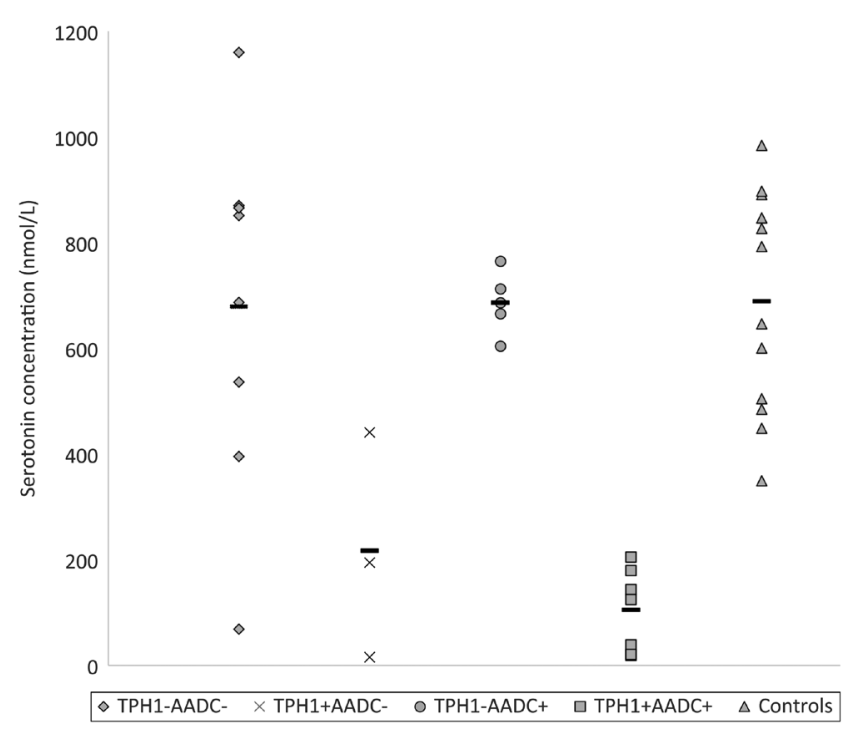

Figure 1

Serum serotonin levels $(\mathrm{nmol} / \mathrm{L})$ in relation to circulating TPH-1 and AADC Aabs. The line represents the mean value of serum serotonin for each patient group and healthy control persons. 
Table 1 Serum levels (nmol/L) of Trp metabolites and L-DOPA in APS-1 according to TPH-1 and AADC Aabs.

\begin{tabular}{|c|c|c|c|}
\hline & TPH1 - AADC $-n=8$ & TPH1+ AADC $-n=3$ & TPH1 - AADC $+n=5$ \\
\hline Trp & $53,527 \pm 8439$ & $56,339 \pm 6241$ & $58,618 \pm 9356$ \\
\hline $5-\mathrm{HT}$ & $679 \pm 339$ & $217 \pm 214^{a}$ & $686 \pm 59$ \\
\hline HIAA & $63 \pm 25$ & $45 \pm 34$ & $69 \pm 17$ \\
\hline $\mathrm{AA}$ & $11 \pm 7$ & $19 \pm 10$ & $12 \pm 5$ \\
\hline HAA & $30 \pm 6$ & $37 \pm 17$ & $32 \pm 11$ \\
\hline L-DOPA & $12 \pm 2$ & $12 \pm 4$ & $14 \pm 4^{c}$ \\
\hline KYN & $1157 \pm 140$ & $1354 \pm 732$ & $1498 \pm 335$ \\
\hline $\mathrm{HK}$ & $28 \pm 9$ & $48 \pm 39$ & $41 \pm 31$ \\
\hline KA & $60 \pm 032$ & $58 \pm 29$ & $54 \pm 20$ \\
\hline IAA & $1242 \pm 316$ & $2019 \pm 1570$ & $1870 \pm 1001$ \\
\hline NAM & $149 \pm 80$ & $104 \pm 56$ & $182 \pm 148$ \\
\hline
\end{tabular}

\begin{tabular}{c}
\hline TPH1+ AADC $+\boldsymbol{n}=\mathbf{7}$ \\
\hline $54,677 \pm 9958$ \\
$105 \pm 78^{b}$ \\
$51 \pm 46$ \\
$15 \pm 6$ \\
$35 \pm 17$ \\
$16 \pm 4^{d}$ \\
$1459 \pm 580$ \\
$42 \pm 21$ \\
$80 \pm 77$ \\
$1484 \pm 407$ \\
$128 \pm 54$
\end{tabular}

\begin{tabular}{c}
\hline Controls $\boldsymbol{n}=\mathbf{1 2}$ \\
\hline $58,422 \pm 7036$ \\
$689 \pm 210$ \\
$35 \pm 16$ \\
$17 \pm 7$ \\
$37 \pm 9$ \\
$9 \pm 3$ \\
$1275 \pm 208$ \\
$34 \pm 8$ \\
$69 \pm 20$ \\
$1587 \pm 429$ \\
$135 \pm 69$
\end{tabular}

\begin{tabular}{l} 
ANOVA \\
\hline$P=0.68$ \\
$P<0.0001$ \\
$P=0.13$ \\
$P=0.25$ \\
$P=0.70$ \\
$P=0.002$ \\
$P=0.44$ \\
$P=0.45$ \\
$P=0.81$ \\
$P=0.35$ \\
$P=0.72$
\end{tabular}

$P$ values for the Dunnett post hoc test comparing patient groups to the control group: ${ }^{a} 0.0085,{ }^{b} P<0.0001, c 0.026$, 0.001 . eL-DOPA: only 11 controls.

\section{Symptoms of depression do not correlate with low 5-HT serum levels in APECED patients}

The complete RBDI questionnaires of 24 (65\%) APECED patients (18 women; mean age 43 years) yielded a mean total RBDI score of 3.4 (range $0-10$ ). Nine patients scored $>5$ - 5 had mild depression $(20.8 \%$, score $5-7)$, while four patients suffered from moderate depression $(16.6 \%$, score 8-15). None had severe depressive symptoms (score $>15$ ). Three patients reported taking oral anti-depressive treatment on a regular basis but their RBDI mean score did not differ from that of patients not on anti-depressive medication. The highest scores were obtained for the questions regarding sleeping disturbances (mean score

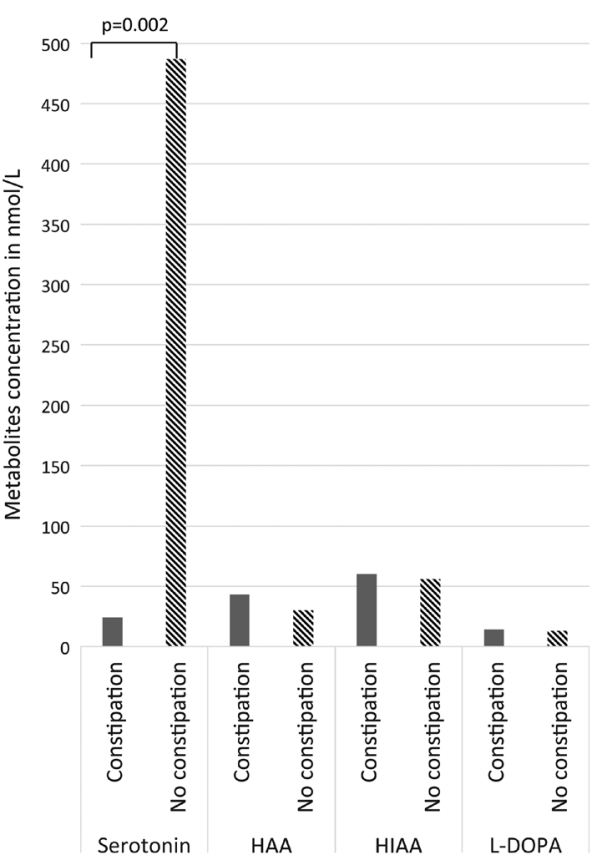

Figure 2

Serum metabolite concentrations ( $\mathrm{nmol} / \mathrm{L}$ ) according to the presence of severe constipation.
0.96 , range $0-3$ ) and fatigue and exhaustion (mean score 0.64 , range $0-3)$. Ten patients (42\%) reported sleeping disturbances, 13 (54\%) suffered from fatigue, and $15(60 \%)$ reported having problems with at least one of either. Neither RBDI total score nor fatigue nor sleep disorder score were correlated to metabolite serum levels (Spearman rho $P>0.05$, data not shown). The use of antidepressants by three patients at the time of sampling had no significant impact on the metabolite levels.

\section{Members of the Escherichia/Shigella genus correlate with APECED status}

Alpha diversity analyses did not produce statistically significant results for either inverse Simpson or Shannon diversity indices when comparing controls to APECED subjects (inverse Simpson $P$ value $=0.21$, Shannon $P$ value $=0.29$; Fig. 3 ). Beta diversity analysis with NMDS using a Bray-Curtis dissimilarity matrix did not show any indication of differences between healthy control persons and APECED cases (data not shown). Using the Bray-Curtis dissimilarity matrix with the adonis implementation of PERMANOVA produced a $P$ value of 0.22 . These last two approaches together suggest that there are no meaningful microbial differences between the two groups at the beta diversity level.

The DNA amplicon sequencing of the $\mathrm{V} 3-\mathrm{V} 4$ regions of the 16S rRNA bacterial gene produced 3662 OTUs distributed into 232 genera, 91 families, 46 orders, 27 classes and 14 phyla. The five most abundant genera in the data set, in decreasing order, are Bacteroides, Prevotella, Alistipes, Ruminococcus and Roseburia (Fig. 4). The five most abundant families are Bacteroidaceae, Ruminococcaceae, Lachnospiraceae, Prevotellaceae and Porphyromonadaceae. The most abundant phyla are Bacteroidetes, Firmicutes, Proteobacteria, Actinobacteria

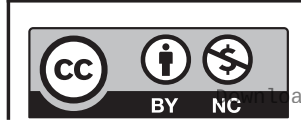

This work is licensed under a Creative Commons Attribution-NonCommercial 4.0 International License. ded from Bioscientifica.com at 04/26/2023 12:52:44PM via free access 

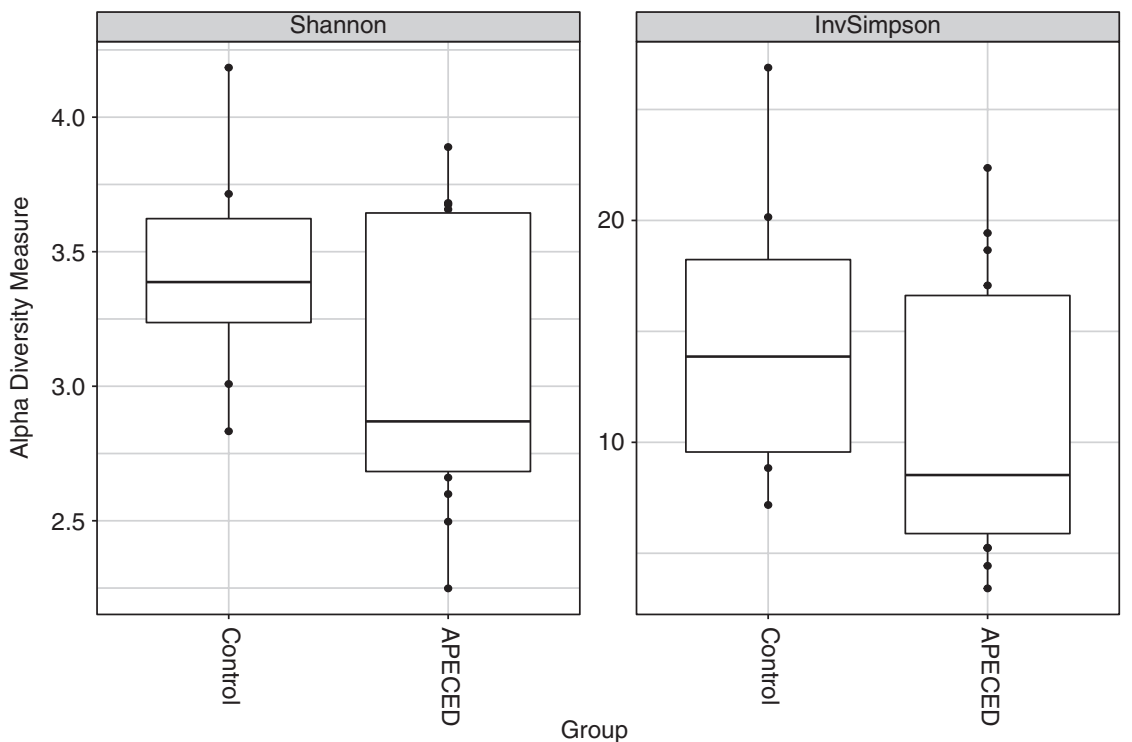

\section{Figure 3}

Stool microbiome alpha diversity indices for control persons and APECED subjects. Based on non-rarefied count data at the OTU level. and Verrucomicrobia. Differential abundance analysis between control persons and APECED subjects was performed at OTU and genus levels, adjusting during modeling for the presence/absence of constipation and diarrhea. Two statistically significant results were produced at both the OTU and the genus level (Table 2) for comparisons between control and APECED subjects. The Enterobacteriaceae OTtu0038 is most probably a false positive, given the sensitivity to the presence of a clear outlier and its overall low mean abundance. It also shows a negative correlation with APECED status. Haemophilus could represent a true positive, but this result should be taken with skepticism given the very low mean abundance of this taxon. Finally, Escherichia/Shigella was detected both at the OTU and genus levels (Fig. 5), with the results being robust and showing an overrepresentation of this taxon in the APECED group. Given the very small number of subjects with constipation (4) and/or diarrhea (7), we did not pursue investigation into possible associations between the latter and taxon abundances, to avoid reporting spurious results.

\section{Discussion}

GI involvement is one of the numerous manifestations of APECED (23). The patients frequently suffer from intermitting periods of diarrhea and severe constipation.

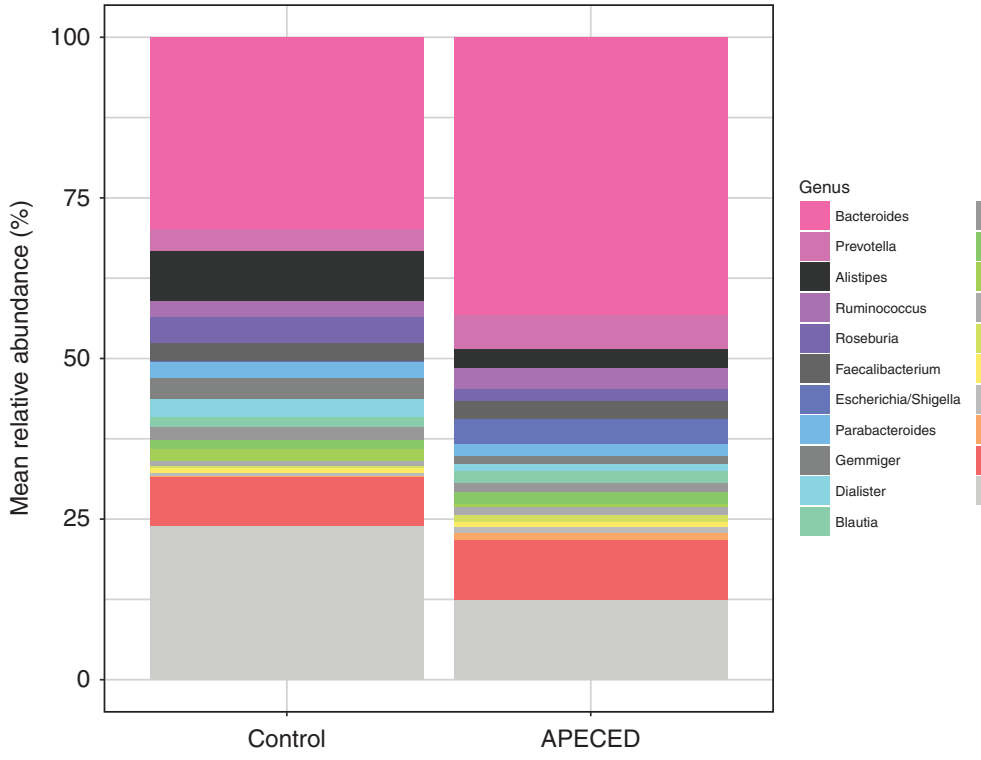

Figure 4

Taxon relative abundances (\%) of the control and APECED groups. The 20 most common genera in the entire data set are explicitly shown. https://ec.bioscientifica.com https://doi.org/10.1530/EC-18-0513 (c) 2019 The authors Published by Bioscientifica Ltd
Barnesiella Clostridium_XIVa Clostridium_XVIII Bifidobacterium Akkermansia Ruminococcus2 Clostridium_ other taxa unclassified tax 


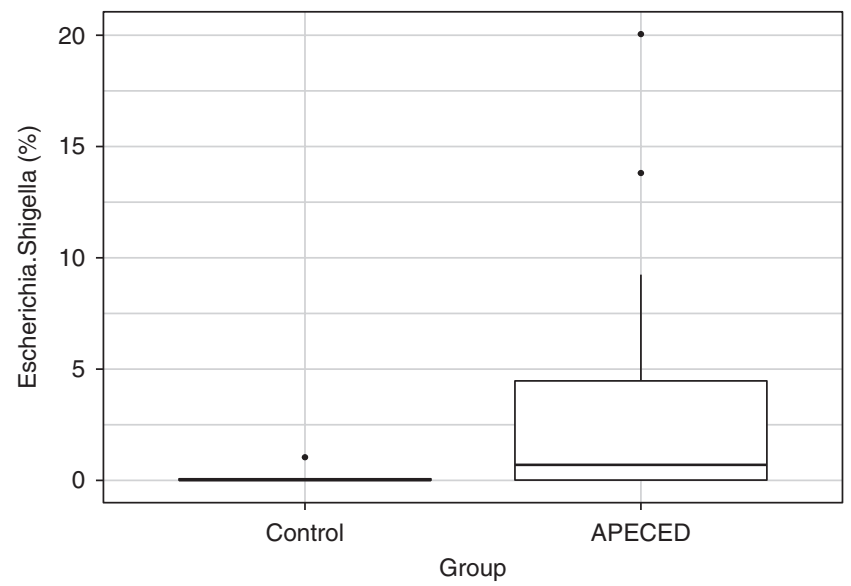

Figure 5

Relative abundances (\%) of the genus Escherichia/Shigella for control persons and APECED subjects.

These symptoms may reflect serum calcium level variation, intestinal infection or autoimmunity (23). Malabsorption affects up to 25\% of the European patients with APECED (23), and up to 80\% in an American cohort (2). It has been shown that patients with APECED typically have a lack of EE cells in the GI tract, which is associated with a lack of expression of chromogranin A and 5-HT $(9,10,12)$. We previously observed that severe constipation symptoms were associated with the lack of 5-HT expression in the intestinal tract (9) and Scarpa and coworkers reported that 5-HT serum levels were significantly lower in APECED patients with anti-TPH Aabs and GI dysfunction (12).

This study shows that serum 5-HT levels tend to be significantly lower in APECED patients (12) and that constipation significantly associates with such low levels. We also confirm that TPH-1 Aabs associate with a decrease in serum 5-HT levels in APECED patients. Interestingly, the presence of AADC Aabs alone did not associate with lowered 5-HT levels, but the presence of both TPH-1 and AADC antibodies seemed to result in even lower 5-HT levels. Also, elevated L-DOPA levels correlated with AADC antibodies. Since AADC catalyzes the conversion of L-DOPA to dopamine, this finding might suggest a functional activity of the AADC Aabs. In our previous study, we found that AADC Aabs associated with constipation (9). However, the present study did not include all patients from the previous cohort. As a result, the small number of patients reporting constipation and diarrhea at the time of blood sampling prevented us to perform statistical comparisons between the various profiles of circulating autoantibodies, symptoms and metabolite levels within the APECED patient group alone.

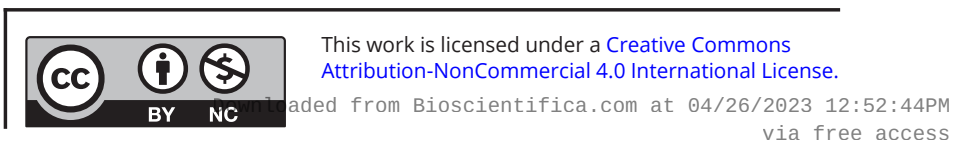


We explored also whether the low serum levels of 5-HT or any other Trp metabolite levels would be associated with symptoms of depression but no such correlation with the RBDI score was found. One may discuss whether the crosssectional metabolite level determination would reflect constant levels and whether the evaluation of depression symptoms with one modified BDI questionnaire, the RBDI, would have needed an evaluation of cognitive function or broad neuropsychological performances.

Nutrients and GI microbiota have an impact on 5-HT metabolism. Spore-forming bacteria, Clostridium spp especially, produce short-chain fatty acids and tryptamine by decarboxylation of tryptophan, which influence TPH-1 expression, 5-HT synthesis and/or 5-HT release $(11,24)$. In turn, it is not known whether 5-HT released into the intestinal lumen exerts any direct impact on gut microbiota composition $(11,25)$. Indeed, as we have reported earlier, APECED patients may also develop Aabs against enteric defensins and often lack Paneth cells (26). Besides, APECED patients also have Aabs against other intestinal cell targets like Villin and AIE-75 (9), which are typically found in IPEX autoimmune enteropathy (27).

The results of the present small-scale exploratory study on possible connections between the gut microbiota and APECED, suggest that either the genus Escherichia or Shigella, as well as Haemophilus, may be overrepresented in APECED. Sequencing a small section of the 16S rRNA gene does not permit to reliably distinguish between Escherichia and Shigella, which in any case is a taxonomic distinction with little biological and evolutionary validity, although of obvious clinical interest. However, given that virtually all sequences in this study that were classified as Escherichia/Shigella belong to the same OTU detected during OTU-level analysis (OTU0006), this OTU probably represents E. coli. Regarding alpha diversity, it should be noticed that, although statistical significance was not reached, the effect estimate is highly suggestive of a drop in the microbial diversity in the APECED population when compared to controls. It is possible that the lack of statistical significance is a false-negative result produced by the small sample size, and therefore, lack of statistical power. The putative lower diversity in the APECED disease state is in agreement with results from previous studies of microbiome and disease, which tend to show lower alpha diversity estimations for disease states in general. All these results should, nevertheless, be regarded as tentative, given the aforementioned small sample size of the microbiome part of this study, and need validation in the form of future studies. The small number of patients and controls constitutes a significant limitation of the present study, and therefore, it can be considered only exploratory in nature. This holds particularly true for the microbiome analysis.

In conclusion, we observed very low levels of circulating 5-HT in APECED patients to be associated with constipation, but not with mental symptoms. Hence, it is recommended to determine serum serotonin as well as TPH1 and AADC Aabs in APECED patients presenting with GI symptoms. Since this study included only Finnish APECED patients with a common AIRE mutation, our observations need to be confirmed in a larger international setting.

\section{Supplementary data}

This is linked to the online version of the paper at https://doi.org/10.1530/ EC-18-0513.

\section{Declaration of interest}

The authors declare that there is no conflict of interest that could be perceived as prejudicing the impartiality of the research reported.

\section{Funding}

Emmi Naskali, Nicolas Kluger, Annamari Ranki, Kai Krohn Helsinki University Hospital Research Funds (TYH 2013235), Finska Läkaresällskapet, Katja Dettmer, Peter J Oefner: INTERREG V BYCZ118.

\section{Acknowledgements}

The authors thank the patients and families with APECED for their cooperation and willingness to participate in our study, as well as the Finnish APECED and Addison registered association (APECED ja Addison Ry, http://www.apeced.org). They thank Alli Tallqvist for her invaluable help and technical assistance.

\section{References}

1 Perheentupa J. Autoimmune polyendocrinopathy-candidiasisectodermal dystrophy. Journal of Clinical Endocrinology and Metabolism 200691 2843-2850. (https://doi.org/10.1210/jc.2005-2611)

2 Ferre EM, Rose SR, Rosenzweig SD, Burbelo PD, Romito KR, Niemela JE, Rosen LB, Break TJ, Gu W, Hunsberger S, et al. Redefined clinical features and diagnostic criteria in autoimmune polyendocrinopathy-candidiasis-ectodermal dystrophy. JCI Insight 20161 e88782. (https://doi.org/10.1172/jci.insight.88782)

3 Orlova EM, Sozaeva LS, Kareva MA, Oftedal BE, Wolff ASB, Breivik L, Zakharova EY, Ivanova ON, Kämpe O, Dedov II, et al. Expanding the phenotypic and genotypic landscape of autoimmune polyendocrine syndrome type 1. Journal of Clinical Endocrinology and Metabolism 2017102 3546-3556. (https://doi.org/10.1210/jc.2017-00139)

4 Zaidi G, Bhatia V, Sahoo SK, Sarangi AN, Bharti N, Zhang L, Yu L, Eriksson D, Bensing S, Kämpe O, et al. Autoimmune polyendocrine syndrome type 1 in an Indian cohort: a longitudinal study. Endocrine Connections 20176 289-296. (https://doi.org/10.1530/EC-17-0022)

5 Kisand K \& Peterson P. Autoimmune polyendocrinopathy candidiasis ectodermal dystrophy. Journal of Clinical Immunology 201535 463-478. (https://doi.org/10.1007/s10875-015-0176-y) 
6 Fishman D, Kisand K, Hertel C, Rothe M, Remm A, Pihlap M, Adler P, Vilo J, Peet A, Meloni A, et al. Autoantibody repertoire in APECED patients targets two distinct subgroups of proteins. Frontiers in Immunology 20178 976. (https://doi.org/10.3389/fimmu.2017.00976)

7 Husebye ES, Gebre-Medhin G, Tuomi T, Perheentupa J, LandinOlsson M, Gustafsson J, Rorsman F \& Kämpe O. Autoantibodies against aromatic L-amino acid decarboxylase in autoimmune polyendocrine syndrome type I. Journal of Clinical Endocrinology and Metabolism 199782 147-150. (https://doi.org/10.1210/ jcem.82.1.3647)

8 Dal Pra C, Chen S, Betterle C, Zanchetta R, McGrath V, Furmaniak J $\&$ Rees Smith B. Autoantibodies to human tryptophan hydroxylase and aromatic L-amino acid decarboxylase. European Journal of Endocrinology 2004150 313-321. (https://doi.org/10.1530/ eje.0.1500313)

9 Kluger N, Jokinen M, Lintulahti A, Krohn K \& Ranki A. Gastrointestinal immunity against tryptophan hydroxylase-1, aromatic L-amino-acid decarboxylase, AIE-75,villin and Paneth cells in APECED. Clinical Immunology 2015158 212-220. (https://doi. org/10.1016/j.clim.2015.03.012)

10 Posovszky C, Lahr G, von Schnurbein J, Buderus S, Findeisen A, Schröder C, Schütz C, Schulz A, Debatin KM, Wabitsch M, et al. Loss of EE cells in autoimmune-polyendocrine-candidiasis-ectodermaldystrophy (APECED) syndrome with gastrointestinal dysfunction. Journal of Clinical Endocrinology and Metabolism 201297 E292-E300. (https://doi.org/10.1210/jc.2011-2044)

11 Spohn SN \& Mawe GM. Non-conventional features of peripheral serotonin signalling - the gut and beyond. Nature Reviews Gastroenterology and Hepatology 201714 412-420. (https://doi. org/10.1038/nrgastro.2017.51)

12 Scarpa R, Alaggio R, Norberto L, Furmaniak J, Chen S, Smith BR, Masiero S, Morlin L, Plebani M, De Luca F, et al. Tryptophan hydroxylase autoantibodies as markers of a distinct autoimmune gastrointestinal component of autoimmune polyendocrine syndrome type 1. Journal of Clinical Endocrinology and Metabolism 201398 704-712. (https://doi.org/10.1210/jc.2012-2734)

13 Kluger N, Jokinen M, Krohn K \& Ranki A. What is the burden of living with autoimmune polyendocrinopathy candidiasis ectodermal dystrophy (APECED) in 2012? A health-related quality-of-life assessment in Finnish patients. Clinical Endocrinology 201379 134-141. (https://doi.org/10.1111/cen.12087)

14 Haase S, Haghikia A, Wilck N, Müller DN \& Linker RA. Impacts of microbiome metabolites on immune regulation and autoimmunity. Immunology 2018154 230-238. (https://doi.org/ 10.1111/ imm.12933)

15 Rieder R, Wisniewski PJ, Alderman BL \& Campbell SC. Microbes and mental health: a review. Brain, Behavior, and Immunity 201766 9-17. (https://doi.org/ 10.1016/j.bbi.2017.01.016)

16 Zhu W, Stevens AP, Dettmer K, Gottfried E, Hoves S, Kreutz M, HollerE, Canelas AB, Kema I \& Oefner PJ. Quantitative profiling of tryptophan metabolites in serum, urine, and cell culture supernatants by liquid chromatography-tandem mass spectrometry. Analytical and Bioanalytical Chemistry 2011401 3249-3261. (https:// doi.org/10.1007/s00216-011-5436-y)

17 Pereira PAB, Aho VTE, Paulin L, Pekkonen E, Auvinen P \& Scheperjans F. Oral and nasal microbiota in Parkinson's disease. Parkinsonism and Related Disorders 201738 61-67. (https://doi. org/10.1016/j.parkreldis.2017.02.026)

18 Andrews S. FastQC: a quality control tool for high throughput sequence data. Cambridge, UK: Babraham Bioinformatics, 2018. (available at: http://www.bioinformatics.babraham.ac.uk/projects/ fastqc/)

19 Martin M. Cutadapt removes adapter sequences from highthroughput sequencing reads. EMBnet.journal 201117 10-12. (https://doi.org/10.14806/ej.17.1.200)

20 Schloss PD, Westcott SL, Ryabin T, Hall JR, Hartmann M, Hollister EB, Lesniewski RA, Oakley BB, Parks DH, Robinson CJ, et al. Introducing mothur: open-source, platform-independent,community-supported software for describing and comparing microbial communities. Applied and Environmental Microbiology 200975 7537-7541. (https:// doi.org/10.1128/AEM.01541-09)

21 McMurdie PJ \& Holmes S. phyloseq: an R package for reproducible interactive analysis and graphics of microbiome census data. PLoS One 20138 e61217. (https://doi.org/10.1371/journal.pone.0061217)

22 Love MI, Huber W \& Anders S. Moderated estimation of fold change and dispersion for RNA-seq data with DESeq2. Genome Biology 2014 15 550. (https://doi.org/10.1186/s13059-014-0550-8)

23 Kluger N, Jokinen M, Krohn K \& Ranki A. Gastrointestinal manifestations in APECED syndrome. Journal of Clinical Gastroenterology 201347 112-120. (https://doi.org/10.1097/ MCG.0b013e31827356e1)

24 Blander JM, Longman RS, Iliev ID, Sonnenberg GF \& Artis D. Regulation of inflammation by microbiota interactions with the host. Nature Immunology 201718 851-860. (https://doi.org/10.1038/ ni.3780)

25 Dodd D, Spitzer MH, Van Treuren W, Merrill BD, Hryckowian AJ, Higginbottom SK, Le A, Cowan TM, Nolan GP, Fischbach MA, et al. A gut bacterial pathway metabolizes aromatic amino acids into nine circulating metabolites. Nature 2017551 648-652. (https://doi. org/10.1038/nature24661)

26 Dobeš J, Neuwirth A, Dobešová M, Vobořil M, Balounová J, Ballek O, Lebl J, Meloni A, Krohn K, Kluger N, et al. Gastrointestinal autoimmunity associated with loss of central tolerance to enteric $\alpha$-defensins. Gastroenterology 2015149 139-150. (https://doi. org/10.1053/j.gastro.2015.05.009)

27 Chida N, Kobayashi I, Takezaki S, Ueki M, Yamazaki Y, Garelli S Scarpa R, Horikawa R, Yamada M, Betterle C, et al. Disease specificity of anti-tryptophan hydroxylase-1 and anti-AIE-75 autoantibodies in APECED and IPEX syndrome. Clinical Immunology 2015156 36-42. (https://doi.org/10.1016/j.clim.2014.10.010)

Received in final form 24 December 2018

Accepted 4 January 2019

Accepted Preprint published online 4 January 2019 https://ec.bioscientifica.com https://doi.org/10.1530/EC-18-0513
(C) 2019 The authors Published by Bioscientifica Ltd

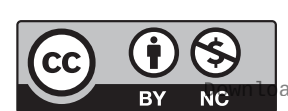

This work is licensed under a Creative Commons Attribution-NonCommercial 4.0 International License. ded from Bioscientifica.com at 04/26/2023 12:52:44PM 